Article

\title{
Unified Description of Dark Energy and Dark Matter within the Generalized Hybrid Metric-Palatini Theory of Gravity
}

\author{
Paulo M. Sá (D) \\ Departamento de Física, Faculdade de Ciências e Tecnologia, Universidade do Algarve, Campus de Gambelas, \\ 8005-139 Faro, Portugal; pmsa@ualg.pt \\ Received: 30 April 2020; Accepted: 3 June 2020; Published: 5 June 2020 \\ check for \\ updates
}

\begin{abstract}
The generalized hybrid metric-Palatini theory of gravity admits a scalar-tensor representation in terms of two interacting scalar fields. We show that, upon an appropriate choice of the interaction potential, one of the scalar fields behaves like dark energy, inducing a late-time accelerated expansion of the universe, while the other scalar field behaves like pressureless dark matter that, together with ordinary baryonic matter, dominates the intermediate phases of cosmic evolution. This unified description of dark energy and dark matter gives rise to viable cosmological solutions, which reproduce the main features of the evolution of the universe.
\end{abstract}

Keywords: modified gravity; dark matter; dark energy

\section{Introduction}

General Relativity, proposed by Albert Einstein more than one hundred years ago [1], has been extremely successful in describing gravitational phenomena on a vast range of scales-from the Solar System to the Universe itself-, surpassing, to date, the most demanding experimental tests [2], including the recent detection of elusive gravitational waves [3].

However, notwithstanding this success, theories of gravity going beyond General Relativity have been receiving increased attention for a variety of reasons, among which the search for an alternative explanation to the observed late-time accelerated expansion of the Universe stands out $[4,5]$. The standard-and simplest-explanation for this accelerated expansion, provided by the concordance $(\Lambda \mathrm{CDM})$ model, resorts to a cosmological constant, but it turns out that the theoretical value of this constant, resulting from quantum field theory calculations, is overwhelmingly different from the one required by observations. This is the well known cosmological-constant problem [6,7], which, hopefully, could be avoided within the framework of a modified theory of gravity.

A plethora of alternative theories to Einstein's General Relativity has been considered in the literature (for a comprehensive review on the subject, see, for instance, Reference [8-10]). In this article, we will focus on a specific class of theories, namely, the hybrid metric-Palatini theory of gravity [11] and its generalized version [12,13], which can be seen as extensions of $f(R)$-gravity [14].

In the hybrid metric-Palatini theory of gravity, the usual Einstein-Hilbert action is supplemented with an additional term, $f(\mathcal{R})$, where $\mathcal{R}$ is the Palatini curvature scalar, defined in terms of a metric-independent connection, and $f$ is an arbitrary function [11]. This novel approach, which combines elements from both the metric and Palatini formalisms, gives rise not only to a viable theory of gravity, but also avoids some drawbacks of the original $f(R)$-gravity $[15,16]$.

The hybrid metric-Palatini theory of gravity admits a dynamically equivalent scalar-tensor representation in terms of a long-range scalar field which plays an active role in cosmology, as well as in galactic dynamics, without conflicting with local experiments even if the scalar field is very 
light [11,17,18]. Other issues of cosmological [19-22] and astrophysical [23-26] relevance have also been addressed within this hybrid theory of gravity. In particular, it has been shown that, for an appropriate choice of the potential of the scalar field, solutions accounting for the late-time acceleration of the universe can be obtained within this hybrid theory [11], a result consolidated soon afterward by several other works [17,20-22].

A generalization of the hybrid metric-Palatini theory of gravity can be achieved by considering the action to be an arbitrary function of both the Ricci and Palatini scalars $f(R, \mathcal{R})[12,13]$. It turns out that this theory is dynamically equivalent to a gravitational theory not with one, but two scalar fields [13]. The weak-field limit [27] and black hole solutions [28] have been investigated within the framework of this theory.

Cosmological solutions of this so-called generalized hybrid metric-Palatini theory of gravity, including those showing periods of accelerated expansion of the universe, were studied in the scalar-tensor representation (Einstein frame [13] and Jordan frame [29]), as well as in the geometric representation [30].

The purpose of the present article is to extend these analyses by proposing a unified description of dark matter and dark energy. To this end, we take advantage of the fact that the generalized hybrid metric-Palatini theory of gravity admits a tensor representation with two scalar fields. Then, one can choose a potential for these two scalar fields such that one of them plays the role of dark matter and, together with ordinary baryonic matter, guarantees the existence of a long-lasting matter-dominated era, and the other scalar field plays the role of dark energy, inducing a late-time era of accelerated expansion of the universe.

Let us note that unified descriptions of dark matter and dark energy have also been proposed in other contexts, such as, for instance, quintessence [31], generalized Chaplygin gas [32,33], phantom cosmology [34], string landscape [35,36], Salam-Sezgin six-dimensional supergravity [37,38], k-essence [39], generalized Galileon theories [40], or mimetic gravity [41].

This article is organized as follows. In the next section we briefly present the generalized hybrid metric-Palatini theory of gravity. In Section 3, choosing an appropriate potential for the interacting scalar fields, we derive a simplified set of equations that provide a unified description of dark matter and dark energy. Numerical solutions of these equations are presented in Section 4. Finally, in Section 5, we present our conclusions.

\section{The Generalized Hybrid Metric-Palatini Theory of Gravity}

For the generalized hybrid metric-Palatini theory of gravity the action is given by [12,13]

$$
S=\frac{1}{2 \kappa^{2}} \int d^{4} x \sqrt{-g} f(R, \mathcal{R})
$$

where $g$ is the determinant of the metric $g_{\alpha \beta}, R$ is the usual Ricci scalar, defined in terms of the metric and the Christoffel connection $\Gamma_{\alpha \beta}^{\mu}=\frac{1}{2} g^{\mu v}\left(\partial_{\alpha} g_{\beta v}+\partial_{\beta} g_{\alpha v}-\partial_{\nu} g_{\alpha \beta}\right), \mathcal{R}$ is the Palatini scalar, constructed with the metric and an independent connection $\hat{\Gamma}_{\alpha \beta}^{\mu}$, such that $\mathcal{R}=g^{\alpha \beta}\left(\partial_{\mu} \hat{\Gamma}_{\alpha \beta}^{\mu}-\partial_{\beta} \hat{\Gamma}_{\alpha \mu}^{\mu}+\hat{\Gamma}_{\nu \mu}^{\mu} \hat{\Gamma}_{\alpha \beta}^{\nu}-\right.$ $\hat{\Gamma}_{v \beta}^{\mu} \hat{\Gamma}_{\alpha \mu}^{v}$ ), and $f$ is an arbitrary (but smooth enough) function of the Ricci and Palatini scalars. Matter couples to the metric, but not to the independent connection (for a review on modified theories of gravity in the Palatini approach see Reference [42]). The natural system of units will be adopted throughout this article, implying that $\kappa=\sqrt{8 \pi} / m_{\mathrm{P}}$, where $m_{\mathrm{P}}=1 / \sqrt{G}=1.22 \times 10^{19} \mathrm{GeV}$ is the Planck mass.

As shown in Reference [13], action (1) can be written in the dynamically equivalent form

$$
S=\frac{1}{2 \kappa^{2}} \int d^{4} x \sqrt{-g}\left[\phi R-\frac{3}{2 \xi}(\partial \xi)^{2}-V(\phi, \xi)\right],
$$

where the scalars field $\phi$ and $\xi$ and the potential $V(\phi, \xi)$ are related to function $f$ appearing in action (1). 
Note that this scalar-tensor representation differs significantly from the one admitted by the original hybrid metric-Palatini theory of gravity (for a derivation of the dynamically equivalent scalar-tensor representation of the original theory see Reference [11]). In particular, it contains an extra scalar field, $\xi$, which, as will be seen below, turns out to be crucial in our proposal for a unified description of dark energy and dark matter. Without going into details, we just mention that in the original theory the scalar field is defined as $\phi=d f(\mathcal{R}) / d \mathcal{R}$ [11], while in the generalized version the two scalar fields are defined as $\xi=-\partial f(R, \mathcal{R}) / \partial \mathcal{R}$ and $\phi=-\xi+\partial f(R, \mathcal{R}) / \partial R[13]$.

Performing a conformal transformation $\tilde{g}_{\alpha \beta}=\phi g_{\alpha \beta}$ and redefining the scalar fields as $\phi=$ $\exp (\sqrt{2 / 3} \kappa \tilde{\phi})$ and $\xi=\kappa^{2} \tilde{\zeta}^{2} / 8$, one obtains the above action in the Einstein frame, namely,

$$
S=\int d^{4} x \sqrt{-\tilde{g}}\left[\frac{1}{2 \kappa^{2}} \tilde{R}-\frac{1}{2}(\tilde{\nabla} \tilde{\phi})^{2}-\frac{1}{2} e^{-\sqrt{2 / 3} \kappa \tilde{\phi}}(\tilde{\nabla} \tilde{\xi})^{2}-\tilde{V}(\tilde{\phi}, \tilde{\xi})\right],
$$

where the potential is defined as

$$
\tilde{V}(\tilde{\phi}, \tilde{\zeta})=\frac{1}{2 \kappa^{2}} e^{-\sqrt{8 / 3} \kappa \tilde{\phi}} V\left(e^{\sqrt{2 / 3} \kappa \tilde{\phi}}, \kappa^{2} \tilde{\zeta}^{2} / 8\right)
$$

Such actions, with two interacting scalar fields and non-standard kinetic terms, motivated by alternative theories to Einstein's General Relativity, as well as by supergravity and string theories, have received much attention in the context of two-field inflationary models [43-47]. In this article, we will be interested in later cosmological epochs, well after the inflationary era, proposing a unified description of dark matter and dark energy based on action (3), in which the scalar fields $\tilde{\zeta}$ and $\tilde{\phi}$ play the role of dark matter and dark energy, respectively.

In what follows, to avoid overloading the notation, the tildes in action (3) will be dropped.

\section{Unified Description of Dark Energy and Dark Matter}

Let us now add radiation and ordinary baryonic matter to action (3), both described by a perfect fluid with energy-momentum tensor

$$
T^{\alpha \beta}=p g^{\alpha \beta}+(\rho+p) u^{\alpha} u^{\beta}
$$

where $u^{\alpha}$ denotes the four-velocity of an observer comoving with the fluid; $\rho$ and $p$ are the energy density and pressure of the fluid, respectively, related through the equation-of-state parameter $w=p / \rho$, which takes the value $w_{\mathrm{R}}=1 / 3$ for radiation and $w_{\mathrm{BM}}=0$ for baryonic matter.

The Einstein equations are then

$$
\begin{aligned}
R_{\alpha \beta}-\frac{1}{2} g_{\alpha \beta} R=\kappa^{2}\{ & \nabla_{\alpha} \phi \nabla_{\beta} \phi-\frac{1}{2} g_{\alpha \beta}(\nabla \phi)^{2}+\left[\nabla_{\alpha} \xi \nabla_{\beta} \xi-\frac{1}{2} g_{\alpha \beta}(\nabla \xi)^{2}\right] e^{-\sqrt{2 / 3} \kappa \phi} \\
& \left.-g_{\alpha \beta} V+\left(\rho_{\mathrm{BM}}+\frac{4}{3} \rho_{\mathrm{R}}\right) u_{\alpha} u_{\beta}+\frac{\rho_{\mathrm{R}}}{3} g_{\alpha \beta}\right\}
\end{aligned}
$$

while the equations for the scalar fields $\phi$ and $\xi$ are given by

$$
\begin{aligned}
& \nabla_{\alpha} \nabla^{\alpha} \phi+\frac{\kappa}{\sqrt{6}}(\nabla \xi)^{2} e^{-\sqrt{2 / 3} \kappa \phi}-\frac{\partial V}{\partial \phi}=0 \\
& \nabla_{\alpha} \nabla^{\alpha} \xi-\sqrt{\frac{2}{3}} \kappa \nabla_{\alpha} \phi \nabla^{\alpha} \xi-\frac{\partial V}{\partial \xi} e^{\sqrt{2 / 3} \kappa \phi}=0 .
\end{aligned}
$$


Since current cosmological measurements constrain the present-time value of the curvature density parameter $\Omega_{k}$ to be very small $[48,49]$, a spatially flat universe can be considered without much loss of generality. Therefore, we assume a flat Friedmann-Robertson-Walker metric,

$$
d s^{2}=-d t^{2}+a^{2}(t) d \Sigma^{2}
$$

where $a(t)$ is the scale factor and $d \Sigma^{2}$ is the metric of the three-dimensional Euclidean space.

Taking into account that perfect-fluid energy-momentum conservation yields $\rho_{\mathrm{R}} \propto a^{-4}$ and $\rho_{\mathrm{BM}} \propto a^{-3}$ for radiation and baryonic matter, respectively, we obtain the equations for the scale factor $a(t)$,

$$
\begin{aligned}
& \left(\frac{\dot{a}}{a}\right)^{2}=\frac{\kappa^{2}}{3}\left[\frac{\dot{\phi}^{2}}{2}+\frac{\dot{\xi}^{2}}{2} e^{-\sqrt{2 / 3} \kappa \phi}+V(\phi, \xi)+\rho_{\mathrm{BM} 0}\left(\frac{a_{0}}{a}\right)^{3}+\rho_{\mathrm{R} 0}\left(\frac{a_{0}}{a}\right)^{4}\right], \\
& \frac{\ddot{a}}{a}=-\frac{\kappa^{2}}{3}\left[\dot{\phi}^{2}+\dot{\xi}^{2} e^{-\sqrt{2 / 3} \kappa \phi}-V(\phi, \xi)+\frac{1}{2} \rho_{\mathrm{BM} 0}\left(\frac{a_{0}}{a}\right)^{3}+\rho_{\mathrm{R} 0}\left(\frac{a_{0}}{a}\right)^{4}\right],
\end{aligned}
$$

and for the scalar fields $\phi(t)$ and $\xi(t)$,

$$
\begin{aligned}
& \ddot{\phi}+3 \frac{\dot{a}}{a} \dot{\phi}+\frac{\kappa}{\sqrt{6}} \dot{\zeta}^{2} e^{-\sqrt{2 / 3} \kappa \phi}+\frac{\partial V}{\partial \phi}=0, \\
& \ddot{\xi}+3 \frac{\dot{a}}{a} \dot{\xi}-\sqrt{\frac{2}{3}} \kappa \dot{\phi} \dot{\xi}+\frac{\partial V}{\partial \xi} e^{\sqrt{2 / 3} \kappa \phi}=0,
\end{aligned}
$$

where an overdot denotes a derivative with respect to time $t$ and a subscript 0 denotes the value of a variable at the present time. For the present-time value of the energy densities of radiation and baryonic matter we take, respectively, $\rho_{\mathrm{R} 0}=9.02 \times 10^{-128} m_{\mathrm{P}}^{4}$ and $\rho_{\mathrm{BM} 0}=8.19 \times 10^{-125} m_{\mathrm{P}}^{4}$, having assumed $H_{0} \equiv(\dot{a} / a)_{0}=67 \mathrm{~km} \mathrm{~s}^{-1} \mathrm{Mpc}^{-1}$.

Now, in order to allow for an unified description of dark energy and dark matter that is, at least in broad lines, in agreement with cosmological data, one needs to choose the potential $V(\phi, \xi)$ appropriately. Taking into account that it should be constructed with exponentials of $\phi$ and powers of $\xi^{2}$ (see Equation (4)), our choice is

$$
\begin{aligned}
V(\phi, \xi) & =V_{a} e^{-\frac{\lambda \kappa}{\sqrt{6}} \phi}\left[1+\left(\frac{\xi}{\xi_{a}}\right)^{2}\right] \\
& =V_{a} e^{-\frac{\lambda \kappa}{\sqrt{6}} \phi}+\frac{1}{2} m_{\xi}^{2}(\phi) \xi^{2}
\end{aligned}
$$

where $V_{a}>0, \lambda$, and $\xi_{a}$ are constants, and $m_{\xi}$ denotes the $\phi$-dependent mass of the scalar field $\xi$,

$$
m_{\xi}(\phi)=\sqrt{\frac{2 V_{a}}{\xi_{a}^{2}}} e^{-\frac{\lambda \kappa}{2 \sqrt{6}} \phi} .
$$

Before proceeding with the analysis of the above system of equations, let us point out that in Reference [13] other choices were made for the potential (4). There, in one of the analyzed cases, the potential was chosen to depend exclusively on the scalar field $\phi$ and to be of the quintessential type, namely, $V \propto \exp (-\lambda \kappa \phi / \sqrt{6})$; this ensures a late-time accelerated expansion of the universe, but at the same time requires dark matter to be introduced "by hand" in the standard matter sector. Another potential, depending both on $\phi$ and $\xi$, was also considered, namely, $V \propto \xi^{\lambda} \exp (-\lambda \kappa \phi / \sqrt{6})$; in this case, the existence of a global attractor corresponding to an accelerated solution (for $\lambda<2 \sqrt{3}$ ) was proven, but the universe does not undergo an intermediate phase of matter domination.

In the present article, the analysis of Reference [13] is extended in order to provide a unified description of dark matter and dark energy. This requires an appropriate choice of the interaction 
potential, namely, a choice that guarantees that the scalar field $\phi$ behaves like dark energy, dominating the dynamics of late-time cosmological evolution and yielding an accelerated expansion of the universe, while the scalar field $\xi$ behaves like pressureless dark matter that, together with ordinary baryonic matter, dominates the intermediate phases of cosmic evolution. As will be shown below, this can be accomplished by choosing a potential of the form given by Equation (14), with the appropriate values of the constants $V_{a}, \xi_{a}$, and $\lambda$.

The system of Equations (10)-(13) can be considerably simplified by taking into account that, for $m_{\xi} \gg H$, the scalar field $\xi$ oscillates rapidly around its minimum ${ }^{1}$, behaving like a nonrelativistic dark-matter fluid with equation of state $\left\langle p_{\mathrm{DM}}\right\rangle=0$ [50], where the brackets $\langle\ldots\rangle$ denote the average over an oscillation.

We define the energy density and pressure of the scalar field $\xi$ as

$$
\begin{aligned}
& \rho_{\mathrm{DM}}=\frac{\dot{\xi}^{2}}{2} e^{-\sqrt{2 / 3} \kappa \phi}+\frac{1}{2} m_{\xi}^{2}(\phi) \xi^{2}, \\
& p_{\mathrm{DM}}=\frac{\dot{\xi}^{2}}{2} e^{-\sqrt{2 / 3} \kappa \phi}-\frac{1}{2} m_{\xi}^{2}(\phi) \xi^{2} .
\end{aligned}
$$

This differs from the situation considered in Reference [50] because of the non-standard kinetic and mass terms, both depending explicitly on the scalar field $\phi$. However, since this field changes very slowly in comparison to $\xi$, the approach used in Reference [50] can be applied here.

Let us then multiply Equation (13) by $\dot{\xi}$ and use the above definition of $\rho_{\mathrm{DM}}$ to obtain

$$
\dot{\rho}_{\mathrm{DM}}+3 \frac{\dot{a}}{a} \dot{\xi}^{2} e^{-\sqrt{2 / 3} \kappa \phi}+\frac{(\lambda-2) \kappa}{2 \sqrt{6}} m_{\tilde{\xi}}^{2} \tilde{\xi}^{2} \dot{\phi}+\sqrt{\frac{2}{3}} \kappa \dot{\phi}\left(\rho_{\mathrm{DM}}-\dot{\xi}^{2} e^{-\sqrt{2 / 3} \kappa \phi}\right)=0
$$

Averaging over an oscillation period and taking into account that $\left\langle p_{\mathrm{DM}}\right\rangle=0$ implies $\left\langle\xi^{2}\right\rangle=$ $\rho_{\mathrm{DM}} / m_{\tilde{\xi}}^{2}$ and $\left\langle\dot{\xi}^{2}\right\rangle=\rho_{\mathrm{DM}} e^{\sqrt{2 / 3} \kappa \phi}$, the above equation can be written as

$$
\dot{\rho}_{\mathrm{DM}}+3 \frac{\dot{a}}{a} \rho_{\mathrm{DM}}+\frac{(\lambda-2) \kappa}{2 \sqrt{6}} \rho_{\mathrm{DM}} \dot{\phi}=0,
$$

which yields the solution

$$
\rho_{\mathrm{DM}}=C\left(\frac{a_{0}}{a}\right)^{3} e^{\frac{(2-\lambda) \kappa}{2 \sqrt{6}} \phi}
$$

where $C$ is an integration constant.

As expected, the energy density of dark matter depends on the scale factor as $a^{-3}$. This is a direct consequence of the fact that we have chosen the potential (14) to be quadratic on the scalar field $\xi$; oscillations around the minimum of the potential mimic a nonrelativistic matter fluid with vanishing pressure. But $\rho_{\mathrm{DM}}$ also depends on the field $\phi$, through the exponential factor, reflecting the existence of a direct coupling between the two fields $\phi$ and $\xi$ in action (3) and in the potential (14). As will be shown in Section 4, such dependence has implications on the cosmic evolution, namely, it leads to a non-simultaneous peaking of the energy densities of dark matter and baryonic matter. For a general discussion of a two-scalar-field model for the interaction of dark energy and dark matter see Reference [51].

1 This condition for $m_{\tilde{\xi}}$, which translates into $\xi_{a}^{2} \ll 2 V_{a}(\dot{a} / a)^{-2} \exp \{-\lambda \kappa \phi / \sqrt{6}\}$, is satisfied as long as the value of $\xi_{a}$ in the potential (14) is chosen to be small enough. 
Now, using Equation (20), the system of Equations (10)-(13) can be considerably simplified, yielding:

$$
\begin{aligned}
& \left(\frac{\dot{a}}{a}\right)^{2}=\frac{\kappa^{2}}{3}\left[\frac{\dot{\phi}^{2}}{2}+V_{a} e^{-\frac{\lambda \kappa}{\sqrt{6}} \phi}+\left(\rho_{\mathrm{BM} 0}+C e^{\frac{(2-\lambda) \kappa}{2 \sqrt{6}} \phi}\right)\left(\frac{a_{0}}{a}\right)^{3}+\rho_{\mathrm{R} 0}\left(\frac{a_{0}}{a}\right)^{4}\right], \\
& \ddot{a} \\
& \frac{\ddot{a}}{3}\left[\frac{\kappa^{2}}{3}\left[\dot{\phi}^{2}-V_{a} e^{-\frac{\lambda \kappa}{\sqrt{6}} \phi}+\frac{1}{2}\left(\rho_{\mathrm{BM} 0}+C e^{\frac{(2-\lambda) \kappa}{2 \sqrt{6}} \phi}\right)\left(\frac{a_{0}}{a}\right)^{3}+\rho_{\mathrm{R} 0}\left(\frac{a_{0}}{a}\right)^{4}\right],\right.
\end{aligned}
$$

and

$$
\ddot{\phi}+3 \frac{\dot{a}}{a} \dot{\phi}+\frac{(2-\lambda) \kappa C}{2 \sqrt{6}}\left(\frac{a_{0}}{a}\right)^{3} e^{\frac{(2-\lambda) \kappa}{2 \sqrt{6}} \phi}-\frac{\lambda \kappa V_{a}}{\sqrt{6}} e^{-\frac{\lambda \kappa}{\sqrt{6}} \phi}=0 .
$$

Instead of the comoving time $t$ let us use a new variable $u$ related to the redshift $z$,

$$
u=-\ln (1+z)=-\ln \left(\frac{a_{0}}{a}\right) .
$$

In terms of this new variable, Equation (23) becomes

$$
\phi_{u u}=-\left\{\left[\frac{\ddot{a}}{a}+2\left(\frac{\dot{a}}{a}\right)^{2}\right] \phi_{u}-\frac{\lambda \kappa V_{a}}{\sqrt{6}} e^{-\frac{\lambda \kappa}{\sqrt{6}} \phi}+\frac{(2-\lambda) \kappa C}{2 \sqrt{6}} e^{\frac{(2-\lambda) \kappa}{2 \sqrt{6}} \phi} e^{-3 u}\right\}\left(\frac{\dot{a}}{a}\right)^{-2},
$$

where the subscript $u$ denotes a derivative with respect to $u ;(\dot{a} / a)^{2}$ and $\ddot{a} / a$ are function of $u, \phi$, and $\phi_{u}$ given by

$$
\begin{aligned}
&\left(\frac{\dot{a}}{a}\right)^{2}= 2 \kappa^{2}\left[V_{a} e^{-\frac{\lambda \kappa}{\sqrt{6}} \phi}+\left(\rho_{\mathrm{BM} 0}+C e^{\frac{(2-\lambda) \kappa}{2 \sqrt{6}} \phi}\right) e^{-3 u}+\rho_{\mathrm{R} 0} e^{-4 u}\right]\left(6-\kappa^{2} \phi_{u}^{2}\right)^{-1}, \\
& \frac{\ddot{a}}{a}=\frac{\kappa^{2}}{6}\left\{4 \kappa^{2}\left[V_{a} e^{-\frac{\lambda \kappa}{\sqrt{6}} \phi}+\left(\rho_{\mathrm{BM} 0}+C e^{\frac{(2-\lambda) \kappa}{2 \sqrt{6}} \phi}\right) e^{-3 u}+\rho_{\mathrm{R} 0} e^{-4 u}\right] \phi_{u}^{2}\left(\kappa^{2} \phi_{u}^{2}-6\right)^{-1}\right. \\
&\left.+2 V_{a} e^{-\frac{\lambda \kappa}{\sqrt{6}} \phi}-\left(\rho_{\mathrm{BM} 0}+C e^{\frac{(2-\lambda) \kappa}{2 \sqrt{6}} \phi}\right) e^{-3 u}-2 \rho_{\mathrm{R} 0} e^{-4 u}\right\} .
\end{aligned}
$$

The above Equations (25)-(27), which provide a unified description of dark matter and dark energy within the generalized hybrid metric-Palatini theory of gravity, will be solved numerically in the next section.

For later convenience let us introduce the density parameters for radiation, baryonic matter, dark matter, and dark energy, respectively,

$$
\begin{aligned}
& \Omega_{\mathrm{R}}=\frac{\rho_{\mathrm{R}}}{\rho_{c}}=\frac{\kappa^{2}}{3} \rho_{\mathrm{R} 0} e^{-4 u}\left(\frac{\dot{a}}{a}\right)^{-2}, \\
& \Omega_{\mathrm{BM}}=\frac{\rho_{\mathrm{BM}}}{\rho_{c}}=\frac{\kappa^{2}}{3} \rho_{\mathrm{BM} 0} e^{-3 u}\left(\frac{\dot{a}}{a}\right)^{-2}, \\
& \Omega_{\mathrm{DM}}=\frac{\rho_{\mathrm{DM}}}{\rho_{c}}=\frac{\kappa^{2}}{3} C e^{\frac{(2-\lambda) \kappa}{2 \sqrt{6}} \phi} e^{-3 u}\left(\frac{\dot{a}}{a}\right)^{-2}, \\
& \Omega_{\mathrm{DE}}=\frac{\rho_{\mathrm{DE}}}{\rho_{c}}=\frac{\kappa^{2}}{3}\left[\frac{\phi_{u}^{2}}{2}+V_{a} e^{-\frac{\lambda \kappa}{\sqrt{6}} \phi}\left(\frac{\dot{a}}{a}\right)^{-2}\right],
\end{aligned}
$$

and the effective equation-of-state parameter,

$$
w_{\mathrm{eff}}=\frac{1}{3}\left[1-\Omega_{\mathrm{BM}}-\Omega_{\mathrm{DM}}-\Omega_{\mathrm{DE}}\left(1-3 \frac{p_{\mathrm{DE}}}{\rho_{\mathrm{DE}}}\right)\right]
$$


where $\rho_{c}=\left(3 / \kappa^{2}\right)(\dot{a} / a)^{2}$ is the critical density, and $\rho_{\mathrm{DE}}$ and $p_{\mathrm{DE}}$ are the energy density and pressure of dark energy, respectively, given by

$$
\begin{aligned}
& \rho_{\mathrm{DE}}=\frac{1}{2}\left(\frac{\dot{a}}{a}\right)^{2} \phi_{u}^{2}+V_{a} e^{-\frac{\lambda \kappa}{\sqrt{6}} \phi}, \\
& p_{\mathrm{DE}}=\frac{1}{2}\left(\frac{\dot{a}}{a}\right)^{2} \phi_{u}^{2}-V_{a} e^{-\frac{\lambda \kappa}{\sqrt{6}} \phi} .
\end{aligned}
$$

\section{Numerical Solutions}

Let us now solve Equations (25)-(27) numerically.

Since we want to include in our numerical simulations the transition from a radiation- to a matter-dominated era and, afterward, from a matter- to a dark-energy-dominated one, we choose to start the integration of Equations (25)-(27) at $u_{i}=-30$, corresponding to redshift $z \simeq 10^{13}$ (well inside the radiation-dominated era of the evolution of the universe).

Having verified that the numerical solutions are quite insensitive to the initial values of the scalar field $\phi$ and its derivative, we choose in all simulations $\phi\left(u_{i}\right)=10^{-3} m_{\mathrm{P}}$ and $\phi_{u}\left(u_{i}\right)=10^{-5} m_{\mathrm{P}}$.

We want to guarantee that, at the present time $u_{0}=0$, the energy densities of dark matter and dark energy, as well as radiation and ordinary baryonic matter, are in agreement with current cosmological measurements $[48,49]$. Since these measurements indicate that dark energy and dark matter contribute, at the present time, with about $69 \%$ and $26 \%$, respectively, of the total energy density of the universe, we take $\rho_{\mathrm{DE} 0}=1.13 \times 10^{-123} m_{\mathrm{P}}^{4}$ and $\rho_{\mathrm{DM} 0}=4.25 \times 10^{-124} m_{\mathrm{P}}^{4}$. We should then impose that the constants $\lambda, V_{a}$, and $C$ satisfy the conditions

$$
\begin{aligned}
& \frac{1}{2}\left[\left(\frac{\dot{a}}{a}\right)^{2} \phi_{u}^{2}\right]_{u=u_{0}}+V_{a} e^{-\frac{\lambda \kappa}{\sqrt{6}} \phi_{0}}=\rho_{\mathrm{DE} 0}, \\
& C e^{\frac{(2-\lambda) \kappa}{2 \sqrt{6}} \phi_{0}}=\rho_{\text {DMO }} .
\end{aligned}
$$

Since this can be achieved with just two of the above three constants, we leave $\lambda$ as a free parameter and satisfy these conditions by choosing appropriately $V_{a}$ and $C$. Therefore, to different values of the parameter $\lambda$ will correspond different pictures of cosmic evolution, all of them with the correct present-time values of the density parameters for radiation, $\Omega_{\mathrm{R} 0}$, baryonic matter, $\Omega_{\mathrm{BM} 0}$, dark matter, $\Omega_{\mathrm{DM} 0}$, and dark energy, $\Omega_{\mathrm{DE} 0}$, but with different dynamics in the past. Naturally, we will focus our attention on the values of the parameter $\lambda$ for which these past dynamics are, at least in broad lines, in agreement with cosmological observations.

Let us recall that the constant $\xi_{a}$, determining the mass of the scalar field $\xi$ (see Equation (15)), is constrained by the condition $m_{\xi} \gg H$, required to guarantee that the scalar field $\xi$ oscillates rapidly around its minimum, thus mimicking a pressureless dark-matter fluid. This condition can always be satisfied by choosing a small enough value of $\xi_{a}$.

Our numerical simulations show that, initially, the scalar field $\phi$, which plays the role of dark energy, remains practically constant. Depending on the value of the parameter $\lambda$, at a certain point this field starts evolving, eventually becoming dominant and inducing an accelerated phase of expansion of the universe (see Figure 1).

This behavior of the field $\phi$ implies that, in the earlier phases of evolution, the energy density of dark matter $\rho_{\mathrm{DM}}$ decreases as $a^{-3}$, exactly as ordinary baryonic matter; for later times, depending on the value of $\lambda$, it decreases faster or slower than $a^{-3}$, the exception being the case $\lambda=2$ for which the exponential in Equation (20) is always equal to one. 


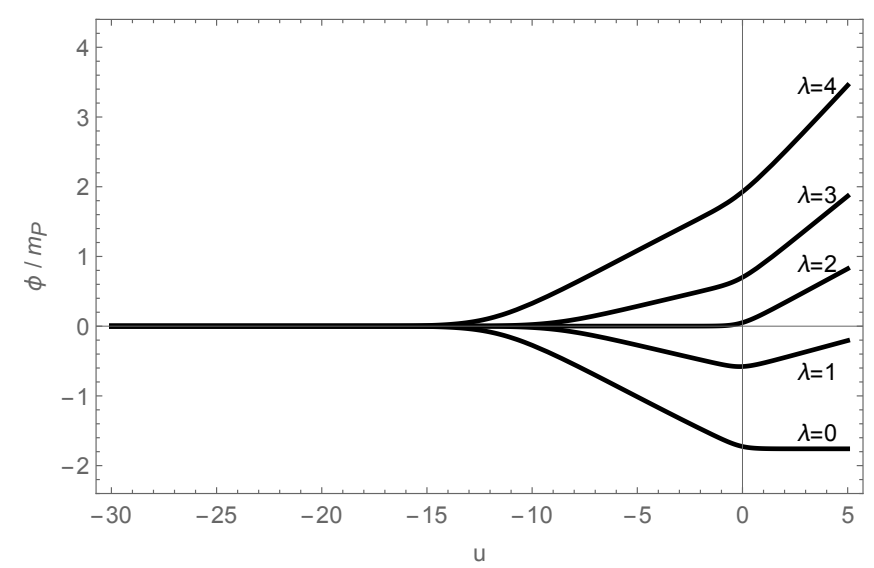

Figure 1. Evolution of the scalar field $\phi$, which plays the role of dark energy, for different values of the parameter $\lambda$. At a certain point, this field becomes dominant and induces an accelerated phase of expansion of the universe. Present time corresponds to $u_{0}=-\ln \left(1+z_{0}\right)=0$.

The different eras of evolution of the universe-radiation dominated, matter dominated, and dark energy dominated-are shown in Figure 2 for the case $\lambda=2$, for which $V_{a}=1.32 \times 10^{-123} \mathrm{~m}_{\mathrm{P}}^{4}$ and $C=4.25 \times 10^{-124} m_{\mathrm{P}}^{4}$ are required in order to satisfy the conditions given by Equations (35) and (36).

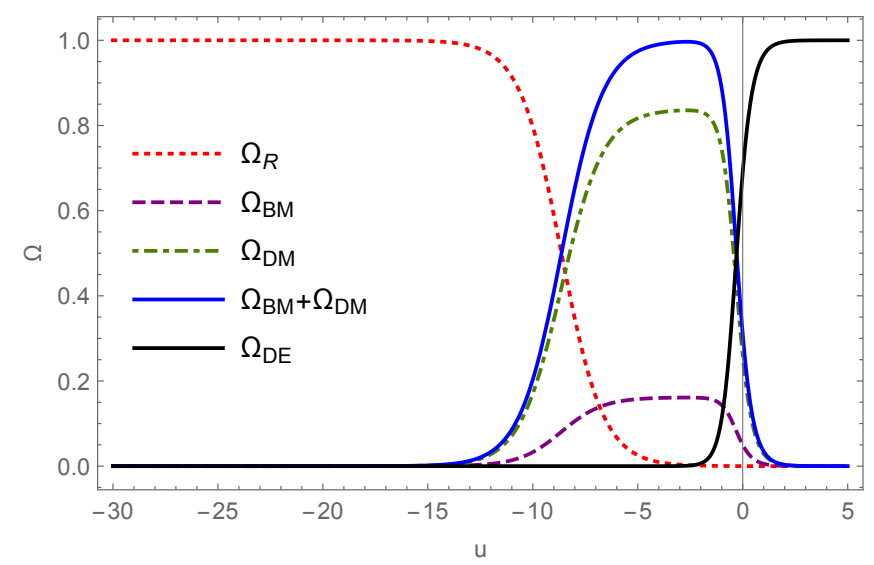

Figure 2. Evolution of the density parameters of radiation, baryonic and dark matter, and dark energy for $\lambda=2$. The transition from a radiation- to a matter-dominated universe occurs well after the primordial nucleosynthesis epoch; the (baryonic and dark) matter-dominated era lasts long enough for structure formation to occur; the transition to a dark-energy-dominated universe takes place in a recent past. At the present time $u_{0}=0$ the density parameters are $\Omega_{\mathrm{DE} 0}=0.69, \Omega_{\mathrm{DM} 0}=0.26, \Omega_{\mathrm{BM} 0}=0.05$, and $\Omega_{\mathrm{R} 0}=5 \times 10^{-5}$.

Initially, the dynamics of the universe is dominated by radiation and $\Omega_{\mathrm{R}} \simeq 1$. Primordial nucleosynthesis, taking place at redshift $z \simeq 10^{8}(u \simeq-18)$, is well inside the radiation-dominated era. At redshift $z \simeq 10^{6}(u \simeq-14)$ begins the transition to a matter-dominated universe and radiation-matter equality is achieved at redshift $z \simeq 10^{4}(u \simeq-9)$. During this matter-dominated era, the energy density of ordinary baryonic matter is a fraction of the energy density of dark matter and $\Omega_{\mathrm{BM}}+\Omega_{\mathrm{DM}} \simeq 1$. Finally, at redshift $z \simeq 1(u \simeq-0.7)$, the scalar field $\phi$ becomes dominant and the transition from a matter- to a dark-energy-dominated universe begins, giving rise to accelerated expansion. At the present time $\left(u_{0}=0\right)$, the density parameters of dark energy, dark matter, baryonic matter, and radiation are, respectively, $\Omega_{\mathrm{DE} 0}=0.69, \Omega_{\mathrm{DM} 0}=0.26, \Omega_{\mathrm{BM} 0}=0.05$, and $\Omega_{\mathrm{R} 0}=5 \times 10^{-5}$. 
The above "history" of the universe reproduces, at least in broad lines, the main features of the observed cosmic evolution. But, naturally, it depends on the value of the parameter $\lambda$ : the more $\lambda$ differs from 2, the more this "history" deviates from the standard one.

These deviations are mainly of three types (see Figure 3 , for the case $\lambda=0$; the case $\lambda=4$ is quite similar).

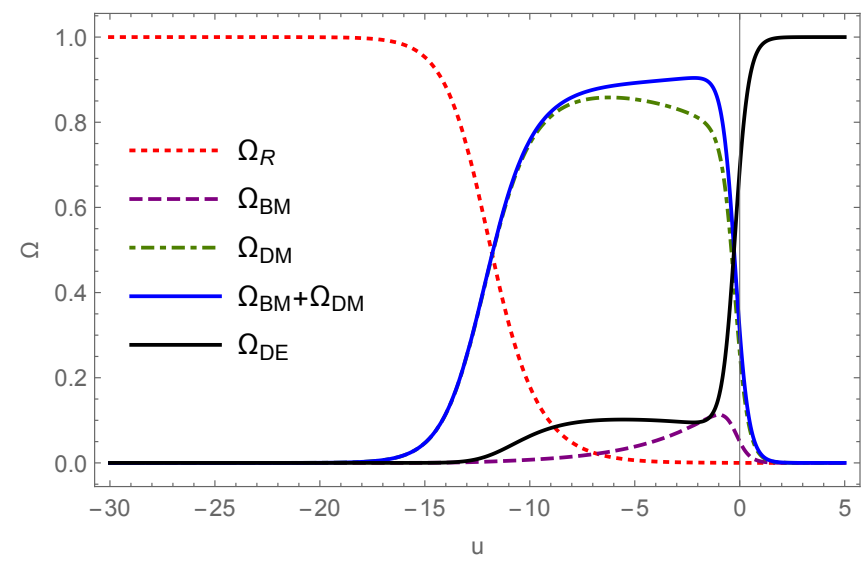

Figure 3. Evolution of the density parameters of radiation, baryonic and dark matter, and dark energy for $\lambda=0$ (similar to the case $\lambda=4$ ). The transition to a matter-dominated universe occurs much earlier than in the case $\lambda=2$, but still after the primordial nucleosynthesis epoch. Contrarily to the case $\lambda=2$, the peaks of $\Omega_{\mathrm{BM}}$ and $\Omega_{\mathrm{DM}}$ do not occur simultaneously. During the matter-dominated era, the energy density of dark energy is already a noticeable fraction of the total energy density. At the present time $u_{0}=0$ the density parameters are $\Omega_{\mathrm{DE} 0}=0.69, \Omega_{\mathrm{DM} 0}=0.26, \Omega_{\mathrm{BM} 0}=0.05$, and $\Omega_{\mathrm{R} 0}=5 \times 10^{-5}$.

First, the transition from a radiation- to a matter-dominated era occurs earlier; our numerical simulations show that, for $|\lambda-2| \gtrsim 2$, this transition take place so early that it enters in conflict with primordial nucleosynthesis. Since this is a conflict one wants to avoid, such values of $\lambda$ should not be considered.

Second, as already mentioned above, for $\lambda \neq 2$ the energy density of dark matter, contrarily to ordinary baryonic matter, does not decrease exactly as $a^{-3}$. It also depends on the scalar field $\phi$ through an exponential factor (see Equation (20)), which arises due to the existence of a direct coupling between the two fields $\phi$ and $\xi$ in action (3) and in the potential (14). This implies that the ratio between the energy densities of baryonic and dark matter is not a constant anymore, but a quantity that depends on the dark energy field, namely, $\rho_{\mathrm{BM}} / \rho_{\mathrm{DM}} \propto \exp \left[\frac{(\lambda-2) \kappa}{2 \sqrt{6}} \phi\right]$. Therefore, for $\lambda \neq 2$ the peaks of $\Omega_{\mathrm{BM}}$ and $\Omega_{\mathrm{DM}}$ do not occur simultaneously.

Third, the dark energy field $\phi$ becomes relevant at earlier stages of the universe's evolution; our numerical simulations show that, for $|\lambda-2| \gtrsim 1$, the energy density of dark energy is already a noticeable fraction of the total energy density during the matter-dominated era. In the case shown in Figure 3 , at redshift $z \simeq 10^{3}(u=-7)$ the energy density of dark energy is already $1 / 9$ of the combined energy densities of dark and baryonic matter.

Let us now analyze the evolution of the effective equation-of-state parameter $w_{\text {eff }}$ (see Figure 4 ), circumscribing ourselves to values of the parameter $\lambda$ that correspond, at least qualitatively, to the observed evolution of the universe, namely, $0 \lesssim \lambda \lesssim 4$.

Initially, for all considered values of $\lambda$, the energy densities of both baryonic and dark matter, as well as dark energy, are negligible, and the dynamics of evolution is dominated by radiation, implying $w_{\text {eff }}=1 / 3$.

Then, at a certain point, matter (baryonic and dark) becomes dominant and the effective equation-of-state parameter decreases gradually from $1 / 3$ to zero (a transition from a radiationto a matter-dominated universe takes place). However, for $|\lambda-2| \gtrsim 1$, this behavior is changed because, during the matter-dominated era, the energy density of dark energy is not negligible anymore, 
contributing noticeably to $w_{\text {eff, }}$ which, therefore, takes a value higher than zero (cases $\lambda=0$ and $\lambda=4$ in Figure 4).

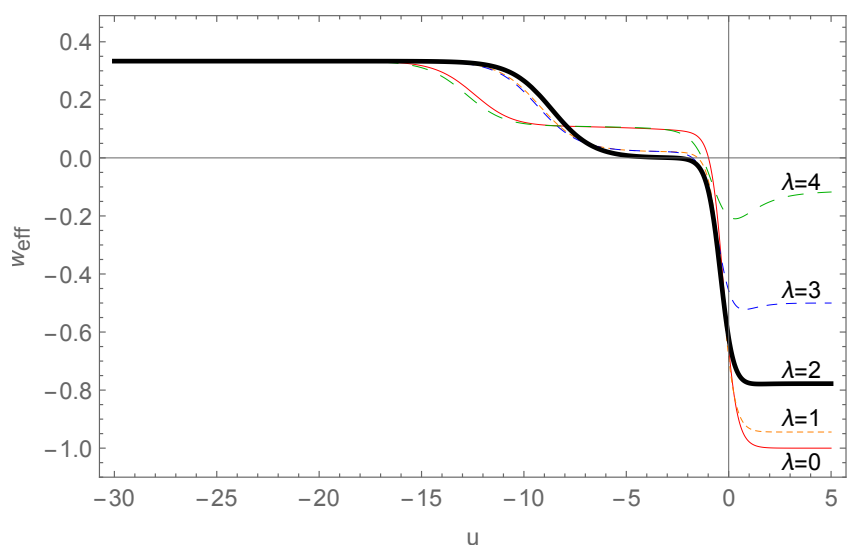

Figure 4. Evolution of the effective equation-of-state parameter $w_{\text {eff }}$ for different values of $\lambda$. Initially, the evolution is dominated by radiation, implying $w_{\text {eff }}=1 / 3$ for all $\lambda$ 's. At certain point, baryonic and dark matter become dominant and $w_{\text {eff }}$ decreases gradually from $1 / 3$ to a near-zero value which depends on $\lambda$. In recent times, dark energy becomes dominant and $w_{\text {eff }}$ assumes negatives values; for $w_{\text {eff }} \leq-1 / 3$ it signals an accelerated expansion of the universe.

Finally, in recent times, the dark energy field $\phi$ becomes dominant and the effective equation-of-state parameter assumes negative values. Asymptotically, $w_{\text {eff }}$ tends to $-1+\lambda^{2} / 18$. Indeed, for negligible $\rho_{\mathrm{R}}, \rho_{\mathrm{BM}}$, and $\rho_{\mathrm{DM}}$, Equations (25)-(27) yield the approximate solution $\phi_{u}=\lambda /(\sqrt{6} \kappa)$ and $V_{a} \exp (-\lambda \kappa \phi / \sqrt{6})=\left(3-\lambda^{2} / 12\right)(H / \kappa)^{2}$, which, upon substitution in Equations (32)-(34), gives $w_{\text {eff }}=-1+\lambda^{2} / 18$. This means that for $\lambda \leq 2 \sqrt{3}$ the universe enters an everlasting period of accelerated expansion. Note, however, that for values of $\lambda$ slightly above $2 \sqrt{3}$ the universe can experience a period of accelerated expansion, but only temporary. For $\lambda=0$, the effective equation-of-state parameter tends asymptotically to the value -1 , which is not unexpected, since in this case the potential given by Equation (14) becomes simply $V(\xi)=V_{a}+m_{\xi}^{2} \xi^{2} / 2$, corresponding to a cosmological constant plus a (dark) matter field.

The above proposed unified description of dark matter and dark energy depends, naturally, on the form of the potential (14); it should be such that one of the scalar fields oscillates around its minimum, mimicking a pressureless dark-matter fluid, and the other field induces a late-time accelerated expansion of the universe, thus behaving like dark energy. Moreover, agreement with current cosmological observations restricts the values of the parameters of the potential, namely, $V_{a}$ should be of the order of $10^{-123} m_{\mathrm{P}}^{4}$, and $\lambda$ should lie in the interval $0 \lesssim \lambda \lesssim 4$. In what concerns the parameter $\xi_{a}$, the condition $m_{\xi} \gg H$ requires it to be much smaller than $10^{-25} m_{\mathrm{P}}$.

\section{Conclusions}

In this article we have proposed a unified description of dark energy and dark matter within the generalized hybrid metric-Palatini theory of gravity.

Working in the scalar-tensor representation of the theory we have shown that, upon an appropriate choice of the potential (see Equation (14)), one of the scalar fields behaves like dark energy, dominating the dynamics of late-time cosmological evolution and yielding an accelerated expansion of the universe, while the other scalar field behaves like pressureless dark matter that, together with ordinary baryonic matter, dominates the intermediate phases of cosmic evolution.

In order to ensure that the picture emerging from our unified description of dark energy and dark matter is in agreement, at least qualitatively, with the standard picture of the universe's evolution, we have imposed that, for each and every solution of Equations (25)-(27), the present-time energy densities—for radiation, ordinary baryonic matter, dark matter, and dark energy—should be equal 
to the measured ones, namely, $\Omega_{\mathrm{DE} 0}=0.69, \Omega_{\mathrm{DM} 0}=0.26, \Omega_{\mathrm{BM} 0}=0.05$, and $\Omega_{\mathrm{R} 0}=5 \times 10^{-5}$. This requirement leaves us with just one free parameter in Equations (25)-(27), $\lambda$, which we could, in principle, choose freely. However, as our numerical simulations show, for $|\lambda-2| \gtrsim 2$, the transition from the radiation- to the matter-dominated era takes place so early that it starts conflicting with primordial nucleosynthesis. Since this is a conflict one wants to avoid, the parameter $\lambda$ was restricted to be in the interval $0 \lesssim \lambda \lesssim 4$.

For such values of $\lambda$, our numerical solutions correspond to universes in which the transition from a radiation- to the matter-dominated era occurs well after primordial nucleosynthesis; the matter-dominated era is long enough to allow, in principle, for structure formation to take place; the transition to an era dominated by dark energy occurs in a recent past (see Figures 2 and 3). Furthermore, for $\lambda \leq 2 \sqrt{3}$, the universe undergoes a late-time everlasting period of accelerated expansion, during which the effective equation-of-state parameter $w_{\text {eff }}$ approaches the value $-1+\lambda^{2} / 18$ (see Figure 4 ). In short, the generalized hybrid metric-Palatini theory of gravity provides a theoretical framework for a unified description of dark matter and dark energy, giving rise to viable cosmological solutions, which reproduce the standard phases of evolution of the universe.

In the present framework, it should be possible, in principle, to extend the analysis backward in time in order to include an inflationary period driven by the scalar field $\phi$ or $\xi$, thus achieving a triple unification of inflation, dark matter, and dark energy within the generalized hybrid metric-Palatini theory of gravity. Indeed, with our choice of the potential $V(\phi, \xi)$ (see Equation (14)), any of the scalar fields $\phi$ or $\xi$ could support an inflationary period; if, then, the decay of this inflaton field were incomplete, the residue could play the role of dark energy or dark matter in the manner proposed in the present article.

In fact, this idea has already been implemented in other contexts. For example, the Salam-Sezgin six-dimensional supergravity theory yields, upon compactification, an action containing two scalar fields $x$ and $y$ with potential $V=A \exp (\sqrt{2} \kappa y)+m_{x}^{2}\left(x-x_{\min }\right)^{2} / 2+\ldots$, where $A$ is a constant related to fundamental quantities of the theory and $m_{x} \propto \exp (\sqrt{2} \kappa y / 2)$ is the $y$-dependent mass of the scalar field $x$; this potential, which is quite similar to ours, allows for a triple unification of inflation, dark matter, and dark energy [38]. As a second example, let us mention the string-landscape inspired model of Reference [35,36], where a unified description of inflation, dark matter, and dark energy was achieved with a single scalar field $\xi$ with a potential $V=V_{a}+m_{\xi}^{2} \xi^{2} / 2$, which is a particular case of ours (for $\lambda=0$ ).

It would be interesting to implement this idea in the context of the generalized hybrid metric-Palatini theory of gravity, allowing for the unification of phenomena apparently as disparate as inflation, dark energy, and dark matter within a single theoretical framework.

Funding: This research received no external funding.

Acknowledgments: The author would like to thank Lucas Sá for useful comments.

Conflicts of Interest: The author declares no conflict of interest.

\section{References}

1. Einstein, A. Die Grundlage der allgemeinen Relativitätstheorie. Ann. Phys. 1916, 49, 769-822. [CrossRef]

2. Will, C.M. The confrontation between General Relativity and experiment. Living Rev. Relativ. 2014, $17,4$. [CrossRef] [PubMed]

3. Abbott, B.P.; et al. [LIGO Scientific Collaboration and Virgo Collaboration]. Observation of gravitational waves from a binary black hole merger. Phys. Rev. Lett. 2016, 116, 061102. [CrossRef]

4. Riess, A.G.; Filippenko, A.V.; Challis, P.; Clocchiatti, A.; Diercks, A.; Garnavich, P.M.; Gilliland, R.L.; Hogan, C.J.; Jha, S.; Kirshner, R.P.; et al. (Supernova Search Team). Observational evidence from supernovae for an accelerating universe and a cosmological constant. Astron. J. 1998, 116, 1009-1038. [CrossRef] 
5. Perlmutter, S.; Aldering, G.; Goldhaber, G.; Knop, R.A.; Nugent, P.; Castro, P.G.; Deustua, S.; Fabbro, S.; Goobar, A.; Groom, D.E.; et al. (The Supernovae Cosmological Project). Measurements of $\Omega$ and $\Lambda$ from 42 high-redshift supernovae. Astrophys. J. 1999, 517, 565-586. [CrossRef]

6. Weinberg, S. The cosmological constant problem. Rev. Mod. Phys. 1989, 61, 1-23. [CrossRef]

7. Martin, J. Everything you always wanted to know about the cosmological constant problem (but were afraid to ask). C. R. Phys. 2012, 13, 566-665. [CrossRef]

8. Berti, E.; Barausse, E.; Cardoso, V.; Gualtieri, L.; Pani, P.; Sperhake, U.; Stein, L.C.; Wex, N.; Yagi, K.; Baker, T.; et al. Testing General Relativity with present and future astrophysical observations. Class. Quantum Grav. 2015, 32, 243001. [CrossRef]

9. Nojiri, S.; Odintsov, S.D.; Oikonomou, V.K. Modified gravity theories on a nutshell: Inflation, bounce and late-time evolution. Phys. Rep. 2017, 692, 1-104. [CrossRef]

10. Heisenberg, L. A systematic approach to generalisations of General Relativity and their cosmological implications. Phys. Rep. 2019, 796, 1-113. [CrossRef]

11. Harko, T.; Koivisto, T.S.; Lobo, F.S.N.; Olmo, G.J. Metric-Palatini gravity unifying local constraints and late-time cosmic acceleration. Phys. Rev. D 2012, 85, 084016. [CrossRef]

12. Flanagan, É.É. Higher-order gravity theories and scalar-tensor theories. Class. Quantum Grav. 2004, 21, 417-426. [CrossRef]

13. Tamanini, N.; Böhmer, C.G. Generalized hybrid metric-Palatini gravity. Phys. Rev. D 2013, 87, 084031. [CrossRef]

14. Sotiriou, T.P.; Faraoni, V. $f(R)$ theories of gravity. Rev. Mod. Phys. 2010, 82, 451-497. [CrossRef]

15. Capozziello, S.; Harko, T.; Koivisto, T.S.; Lobo, F.S.N.; Olmo, G.J. Hybrid metric-Palatini gravity. Universe 2015, 1, 199-238. [CrossRef]

16. Koivisto, T.S.; Tamanini, N. Ghosts in pure and hybrid formalisms of gravity theories: A unified analysis. Phys. Rev. D 2013, 87, 104030. [CrossRef]

17. Capozziello, S.; Harko, T.; Koivisto, T.S.; Lobo, F.S.N.; Olmo, G.J. Cosmology of hybrid metric-Palatini $f(X)$-gravity. J. Cosmol. Astropart. Phys. 2013, 4, 11. [CrossRef]

18. Capozziello, S.; Harko, T.; Koivisto, T.S.; Lobo, F.S.N.; Olmo, G.J. The virial theorem and the dark matter problem in hybrid metric-Palatini gravity. J. Cosmol. Astropart. Phys. 2013, 7, 24. [CrossRef]

19. Böhmer, C.G.; Lobo, F.S.N.; Tamanini, N. Einstein static universe in hybrid metric-Palatini gravity. Phys. Rev. D 2013, 88, 104019. [CrossRef]

20. Lima, N.A. Dynamics of linear perturbations in the hybrid metric-Palatini gravity. Phys. Rev. D 2014, 89, 083527. [CrossRef]

21. Lima, N.A.; Smer-Barreto, V. Constraints on hybrid metric-Palatini gravity from background evolution. Astrophys. J. 2016, 818, 186. [CrossRef]

22. Carloni, S.; Koivisto, T.; Lobo, F.S.N. Dynamical system analysis of hybrid metric-Palatini cosmologies. Phys. Rev. D 2015, 92, 064035. [CrossRef]

23. Capozziello, S.; Harko, T.; Koivisto, T.S.; Lobo, F.S.N.; Olmo, G.J. Galactic rotation curves in hybrid metric-Palatini gravity. Astropart. Phys. 2013, 50, 65-75. [CrossRef]

24. Borka, D.; Capozziello, S.; Jovanović, P.; Borka Jovanović, V. Probing hybrid modified gravity by stellar motion around Galactic Center. Astropart. Phys. 2016, 79, 41-48. [CrossRef]

25. Danilă, B.; Harko, T.; Lobo, F.S.N.; Mak, M.K. Hybrid metric-Palatini stars. Phys. Rev. D 2017, 95, 044031. [CrossRef]

26. Danilă, B.; Harko, T.; Lobo, F.S.N.; Mak, M.K. Spherically symmetric static vacuum solutions in hybrid metric-Palatini gravity. Phys. Rev. D 2019, 99, 064028. [CrossRef]

27. Bombacigno, F.; Moretti, F.; Montani, G. Scalar modes in extended hybrid metric-Palatini gravity: Weak field phenomenology. Phys. Rev. D 2019, 100, 124036. [CrossRef]

28. Rosa, J.L.; Lemos, J.P.S.; Lobo, F.S.N. Stability of Kerr black holes in generalized hybrid metric-Palatini gravity. Phys. Rev. D 2020, 101, 044055. [CrossRef]

29. Rosa, J.L.; Carloni, S.; Lemos, J.P.S.; Lobo, F.S.N. Cosmological solutions in generalized hybrid metric-Palatini gravity. Phys. Rev. D 2017, 95, 124035. [CrossRef]

30. Rosa, J.L.; Carloni, S.; Lemos, J.P.S. Cosmological phase space of generalized hybrid metric-Palatini theories of gravity. Phys. Rev. D 2020, 101, 104056. [CrossRef] 
31. Sahni, V.; Wang, L. New cosmological model of quintessence and dark matter. Phys. Rev. D 2000, 62, 103517. [CrossRef]

32. Bento, M.C.; Bertolami, O.; Sen, A.A. Generalized Chaplygin gas, accelerated expansion, and darkenergy-matter unification. Phys. Rev. D 2002, 66, 43507. [CrossRef]

33. Zhang, X.; Wu, F.-Q.; Zhang, J. New generalized Chaplygin gas as a scheme for unification of dark energy and dark matter. J. Cosmol. Astropart. Phys. 2006, 01, 003. [CrossRef]

34. Capozziello, S.; Nojiri, S.; Odintsov, S.D. Unified phantom cosmology: Inflation, dark energy and dark matter under the same standard. Phys. Lett. B 2006, 632, 597-604. [CrossRef]

35. Liddle A.R.; Ureña-López, L.A. Inflation, dark matter, and dark energy in the string landscape. Phys. Rev. Lett. 2006, 97, 161301. [CrossRef]

36. Liddle, A.R.; Pahud, C.; Ureña-López, L.A. Triple unification of inflation, dark matter, and dark energy using a single field. Phys. Rev. D 2008, 77, 121301(R). [CrossRef]

37. Anchordoqui, L.; Goldberg, H.; Nawata, S.; Nuñez, C. Cosmology from string theory. Phys. Rev. D 2007, 76, 126005. [CrossRef]

38. Henriques, A.B.; Potting, R.; Sá, P.M. Unification of inflation, dark energy, and dark matter within the Salam-Sezgin cosmological model. Phys. Rev. D 2009, 79, 103522. [CrossRef]

39. Bose, N.; Majumdar, A.S. k-essence model of inflation, dark matter, and dark energy. Phys. Rev. D 2009, $79,103517$. [CrossRef]

40. Koutsoumbas, G.; Ntrekis, K.; Papantonopoulos, E.; Saridakis, E.N. Unification of dark matter-dark energy in generalized Galileon theories. J. Cosmol. Astropart. Phys. 2018, 02, 003. [CrossRef]

41. Dutta, J.; Khyllep, W.; Saridakis, E.N.; Tamanini, N.; Vagnozzi, S. Cosmological dynamics of mimetic gravity. J. Cosmol. Astropart. Phys. 2018, 02, 041. [CrossRef]

42. Olmo, G.J. Palatini approach to modified gravity: $f(R)$ theories and beyond. Int. J. Mod. Phys. D 2011, 20, 413-462. [CrossRef]

43. Berkin, A.L.; Maeda, K. Inflation in generalized Einstein theories. Phys. Rev. D 1991, 44, 1691-1704. [CrossRef] [PubMed]

44. Starobinsky, A.A.; Tsujikawa, S.; Yokoyama, J. Cosmological perturbations from multi-field inflation in generalized Einstein theories. Nucl. Phys. B 2001, 610, 383-410. [CrossRef]

45. Di Marco, F.; Finelli, F.; Brandenberger, R. Adiabatic and isocurvature perturbations for multifield generalized Einstein models. Phys. Rev. D 2003, 67, 063512. [CrossRef]

46. Lalak, Z.; Langlois, D.; Pokorski, S.; Turzyński, K. Curvature and isocurvature perturbations in two-field inflation. J. Cosmol. Astropart. Phys. 2007, 7, 014. [CrossRef]

47. Chakravarty, G.K.; Das, S.; Lambiase, G.; Mohanty, S. Dilaton assisted two-field inflation from no-scale supergravity. Phys. Rev. D 2016, 94, 023521. [CrossRef]

48. Ade, P.A.R.; Aghanim, N.; Arnaud, M.; Ashdown, M.; Aumont, J.; Baccigalupi, C.; Banday, A.J.; Barreiro, R.B.; Bartlett, J.G.; Bartolo, N.; et al. (Planck Collaboration). Planck 2015 results: XIII. Cosmological parameters. Astron. Astrophys. 2016, 594, A13.

49. Aghanim, N.; Akrami, Y.; Ashdown, M.; Aumont, J.; Baccigalupi, C.; Ballardini, M.; Banday, A.J.; Barreiro, R.B.; Bartolo, N.; Basak, S.; et al. (Planck Collaboration). Planck 2018 results: VI. Cosmological parameters. arXiv 2018, arXiv:1807.06209.

50. Turner, M.S. Coherent scalar-field oscillations in an expanding universe. Phys. Rev. D 1983, 28, $1243-1247$. [CrossRef]

51. Bertolami, O.; Carrilho, P.; Páramos, J. Two-scalar-field model for the interaction of dark energy and dark matter. Phys. Rev. D 2012, 86, 103522. [CrossRef]

(C) 2020 by the author. Licensee MDPI, Basel, Switzerland. This article is an open access article distributed under the terms and conditions of the Creative Commons Attribution (CC BY) license (http:/ / creativecommons.org/licenses/by/4.0/). 